\title{
A Study on the Development of Sports Lifestyle of the Elderly in Jiangsu Province
}

\author{
Juhua Song \\ Sports Department \\ Nanjing University of Information Science \& Technology \\ Nanjing, China 210044
}

\begin{abstract}
In this paper, the current situation and development trend of sports lifestyle of urban elderly people in Jiangsu Province are studied by means of literature review, questionnaire and mathematical statistics, aiming to construct healthy and casual sports lifestyle of the elderly in Jiangsu province under the guidance of healthy China. The paper points out the main factors that restrict the sports lifestyle of the elderly in the social process and puts forward the corresponding countermeasures and suggestions to provide the theoretical basis for the scientific guidance and intervention of sports lifestyle of the urban elderly people in Jiangsu Province.
\end{abstract}

Keywords—the elderly; sports lifestyle; development

\section{INTRODUCTION}

In the 21st century, the transformation from traditional society to modern society in China has entered a new stage of development. With the deepening of influence of social transformation on people's lifestyle, people gradually increase the requirements for the quality of life while enjoying the convenience and quick way of life. Thus, sports that can promote the comprehensive development of people's mind and body begin to integrate into people's lives, becoming the content and necessities of life and sports lifestyle come into being. Sports lifestyle as a new lifestyle is an inevitable choice for modern people to adapt to the development of modern society. And it has become an identification of civilized society to let the elderly share the fruits of social development and improve their living quality. In 2000, at the commemoration of the 50th anniversary of International Association of Gerontology in Italy, it is proposed that the ultimate goal of the scientific research of the elderly is to improve their living quality. Physical education theory for the senior also has the same purpose, that is, to improve self-care ability of the elderly, reduce the morbidity of chronic diseases, and improve their living quality.

\section{THE CHARACTERISTICS OF SPORTS LIFESTYLE OF URBAN ELDERLY PEOPLE IN JIANGSU PROVINCE}

The purposes of taking sports as a lifestyle mainly includes promoting health from oneself, entertaining the body and mind, improving living standards, and enriching

Fund Project: Philosophy and Social Science Research Fund Project of Colleges in Jiangsu in 2017

\author{
Jiangyue $\mathrm{Wu}$ \\ Sports Department \\ Nanjing University of Information Science \& Technology \\ Nanjing, China 210044
}

life culture; expressing oneself, extending personality, and release oneself; tapping potential, improve the limit, and working harder. With the development of social economy, restriction of population policy, and renewal of people's life concept, lifestyle of the urban elderly shows the characteristics of modern leisure, voluntary and psychological satisfaction.

\section{A. The Shown Purposefulness}

The key to the establishment of sports lifestyle is that people should form correct sports values, because sports values determine the people's sports behavior. Sports lifestyle of the elderly is the behavior of the elderly regarding sports as the needs and content of life and consciously participating in sports activities under the guidance of certain values. What the goals and attitudes of the elderly on sports and sports activities are and how they understand sports and sports are the sports values. This behavior of consciously participating in sports activities is carried out under the guidance of certain values, therefore, it has purposefulness. The purpose of our advocating of the elderly to actively participate in sports activities is to let them enjoy sports, improve the living quality and promote the harmonious development of human. The key of emphasizing the harmonious development of human is improvement of lifestyle. We should develop and improve the sports concept awareness of the elderly through a variety of channels to guide their sports behavior, and form and establish a good sports lifestyle.

\section{B. The Shown Regularity}

The elderly can choose different sports lifestyles according to different individuals and different groups, so only by transforming the random and accidental sports behaviors to regular and stable sports behaviors can they have the essential property of sports lifestyle. Therefore, sports lifestyle of the elderly is both multi-level, highlygeneralized and comprehensive concept and specific concept reflected by vivid and specific sports participation form and behavior of a person.

\section{The Shown Consciousness}

We can only let sports become the habit and routine of the elderly's life as well as the necessaries of their life, that is, 
integrating sports into their lives, and making sports become their spontaneous demand and conscious behavior without any mandatory, so they can independently choose the content and form sports activities based on a variety of objective conditions. Social development has brought the transformation of sports values of the elderly. After the elderly having a correct understanding of sports fitness, this positive values will dominate their corresponding sports behavior, serving as the impetus of their they active participation in various daily sports activities. From spontaneous to conscious, sports achieve the unity as a means as well as a purpose.

\section{THE CURRENT SitUATION OF SPORTS LIFESTYLE OF THE URBAN ELDERLY IN JIANGSU PROVINCE}

\section{A. Diversified Motives of Sports Activities}

Sports have the biological functions of body building, disease prevention and bringing health and longevity to people as well as the function of eliminating trouble, cultivating sentiment, establishing good interpersonal relationships and self-realizing. This is what a lot of old people can realize. In addition, the survey found that the main motive of the elderly to participate in sports activities is to find spiritual sustenance. Such elderly people account for $75.8 \%$ of the elderly sports enthusiasts, following the elderly who have the motives of body building and longevity, which shows that sports not only are part of the elderly's lives, but also become their spiritual pillar. After the retirement, the elderly lack exchanges between the past colleagues and the majority of children in the family have grown up to "build another home". The widowed elderly also account for a certain proportion. Therefore, a sense of loss and loneliness makes some people depressed, fall ill and never recover while more elderly people can adjust the mentality in time, change the way of life and positive participate in various leisure activities. Sports are the effective way for them to strengthen interpersonal relationships and relieve loneliness. $61.3 \%$ of them have this motive. The elderly have some chronic diseases more or less, so currently $86.7 \%$ of the elderly regard the health as the priority. Since some sports activities have been certified by Chinese medicine as the "good recipe" for prevention and treatment of diseases, such as tai chi, and wuqin play and so on, many elderly taking sports activities as the main form of leisure lifestyle not only due to spirit needs, but also because of physiological needs. The elderly with this motive account for $71.4 \%$.

\section{B. High Attendance in Sports Activities}

The frequency of sports activities among the elderly in Jiangsu Province is high. Under normal circumstances, they participate in sports activities every day with many of them taking morning exercise and participating in sports activities of different intensity in the afternoon. Such old people account for $13.9 \%$ of the urban elderly. At the same time, the time when they are engaged in activities is long, with $89 \%$ of the elderly doing more than an hour. Among the elderly with sports activity habit, the people who take three or more times per week and exercise more than one hour each time account for $86.1 \%$. It can be seen that the sports activities have indeed become an indispensable spiritual pillar for the life of the elderly. The elderly who are keen on sports activities generally keep exercising for years, of which $82.9 \%$ have kept for 3 to 5 years while $53.8 \%$ of them have kept for over 6 years. It can be seen that in Jiangsu, the frequency of sports activity of the urban elderly is high, time is long and most of the elderly keep for years.

\section{Establish a Good Relationship with Each Other}

The difference between the elderly and the young people engaging in sports activities is that the activities of the urban elderly in Jiangsu Province are mostly in the form of groups, and often dozens of people with the same interest form a group to do a favorite project together in a certain period of time. Such group once formed, it is not easy to change. The survey also found that, besides the sports activities, the old people often talk about life and politics together, and often concern about national affairs.

\section{Places are Fixed While Activities are Diverse}

The sports events that the urban elderly in Jiangsu Province like to choose are not only the so-called slow pace sports in the past, such as tai chi, boccie, etc., but also some rhythmic events, such as square dance, ballroom dance, golf, and tennis and so on. In the aspect of site, because Jiangsu provincial government attaches importance to the elderly sports activities and there is the public opinion on caring the elderly in our society, some enterprises and institutions are equipped with elderly sports organizations, such as the elderly sports association and so on. The society also invests to build various forms of sports room, sports counseling station (point), and sports center for the elderly, so that they have places to do sports, and have events to do, which also meets the basic needs of the urban elderly in Jiangsu province to do sports.

\section{RESEARCH ON THE DEVELOPMENT OF SPORTS LIFESTYLE OF THE URBAN ELDERLY IN JIANGSU PROVINCE}

Modern society is not only a well-off society where the social structure is more reasonable, human well-being can be reflected, and people are put in the first, but also a "social form that takes person's free and comprehensive development as the basic principles." Therefore, the role of sports in the future society will also be expressed to the greatest extent and people also pay more attention to the demands for sports and idea of sports lifestyle. Sports lifestyle is an important part of the elderly's spiritual lifestyle, which has a great impact on the physical and mental health of the elderly. With the development trend of transformation of population structure in Jiangsu Province and the characteristics of modern urban lifestyle, the sports lifestyle of the urban elderly in Jiangsu Province should have a good development trend.

\section{A. Sports Activity Style Becomes More Scientific}

The urban elderly in Jiangsu Province regard engaging in sports activities as one of the lifestyles, because sports have 
the unique function of both fitness and mental health. The physical and mental characteristics of the elderly determine the difference in doing sports between groups of other ages. Relative departments of Jiangsu provincial government make efforts to speed up the construction of social sports instructors and the establishment of community sports instructor system. The establishment of sports instructor system can make full use of college sports resources. They can deploy some of the physical education teachers to do sports activities counseling for the elderly on a regular basis, so that their sports activities can become more scientific and more colorful. With the supporting services of biological, psychological, social monitoring system, as well as a large number of social sports instructors entering the community, the sports activities of the elderly will become more scientific and "exercise prescription" suitable for the elderly's body and mental health will continue to be revised, affirmed and popularized.

\section{B. Sports Life Become Popular}

Sports life style is the stable form and behavior characteristics formed by individuals or groups participating in sports activities regularly and consciously based on certain objective conditions under the guidance of the values of certain subjects. Human beings at different ages need to overcome various effects of unfavorable environment from their own and the outside world in order to achieve the purposes of physical and mental health and longevity, which requires people to active participate in sports activities in a long period of life to achieve the aims of lifelong sports. The sports life of the urban elderly in Jiangsu province makes their sports run through their life to realize lifelong sports. Therefore, actively carrying out the elderly sports activities is an important part of the implementation of the national fitness program, the effective way to expand the sports population, and the fate of lifelong sports.

\section{Leisure Sports Activities Tend to Comprehensiveness}

With the continuous expansion and extension of role of leisure sports life in urban life, urban life and the connotation of urban humanities continue to evolve, so the lifestyle of the urban elderly is also undergoing a series of changes. Sports leisure creates a relaxed and happy living space from the development and improvement of people to improve the quality of life, serving as the paradise of urban people to enjoy the leisure life. The proportion of the elderly population is growing. In the proportion of the various ages doing the sport, that of the elderly is the largest. With the graduate upgrading of values and view of life, modern urban lifestyles are becoming more and more popular, and leisure sports will also develop towards the direction of popularization, making the sports population increase constantly. At the same time, due to changes in the cultural structure of old-aged friends, their cultural and artistic taste continues to improve, so their entertainment is no longer the simple sport event such as running and boxing, but the comprehensive sports and entertainment activities integrating culture, entertainment and fitness. For example, currently, many urban elderly sports centers have become the comprehensive places integrating sports, culture, and entertainment.

\section{CONCLUSION}

Sports lifestyle in the new era is a scientific, healthy, civilized lifestyle as well as a kind of sports culture. It refers that in the social transformation period, after people are satisfied in the material and cultural life and they want to get another lifestyle, which is not only controlled by the subjective control, but also influenced by the society, politics, economy, and culture and so on. Due to the continuous development of society and changes in population structure, "aging society" is an indisputable fact. Now the lifestyle of the urban elderly in Jiangsu province has greatly changed, and sport has become an indispensable part of their lives, so that it has formed a distinctive sports lifestyle. As the elderly sports population continues to expand and the direction of their cultural and sports activities develops towards integration, the sports lifestyle of the elderly becomes more scientific.

\section{REFERENCES}

[1] Miao Dapei. Discussion on sports lifestyle. Beijing: Beijing Sport University Press. 2004, 90。

[2] Wu Jiangyue. An Empirical Study on the Leisure Sports Behavior of the elderly in Jiangsu province in the process of building a well-off society in an all-round way. Beijing: China Meteorological Press. 2013: 128 。

[3] Xin Li. Current Situation and Development Trend of Sports Lifestyle of the Urban Elderly in China China Sport Science and Technology, 2001(3): 20-21。

[4] Pang Yuanning. On the Current Situation of Sports of Different Age Groups in China. China sport science, 2000(1): 23-25 